\title{
Detection of Mastitis in Dairy Cattle by Use of Mixture Models for Repeated Somatic Cell Scores: A Bayesian Approach via Gibbs Sampling
}

\author{
J. Ødegård, ${ }^{\star}$ J. Jensen,† P. Madsen,† D. Gianola,‡,* \\ G. Klemetsdal, ${ }^{*}$ and B. Heringstad* \\ *Department of Animal Science, Agricultural University of Norway, \\ $\mathrm{N}-1432$ Ås, Norway \\ †Department of Animal Breeding and Genetics, \\ Danish Institute of Agricultural Sciences, \\ Research Centre Foulum, DK-8830 Tjele, Denmark \\ ‡Department of Animal Sciences, University of Wisconsin-Madison, \\ Madison 53706
}

\begin{abstract}
The distribution of somatic cell scores could be regarded as a mixture of at least two components depending on a cow's udder health status. A heteroscedastic two-component Bayesian normal mixture model with random effects was developed and implemented via Gibbs sampling. The model was evaluated using datasets consisting of simulated somatic cell score records. Somatic cell score was simulated as a mixture representing two alternative udder health statuses ("healthy" or "diseased"). Animals were assigned randomly to the two components according to the probability of group membership $\left(P_{m}\right)$. Random effects (additive genetic and permanent environment), when included, had identical distributions across mixture components. Posterior probabilities of putative mastitis were estimated for all observations, and model adequacy was evaluated using measures of sensitivity, specificity, and posterior probability of misclassification. Fitting different residual variances in the two mixture components caused some bias in estimation of parameters. When the components were difficult to disentangle, so were their residual variances, causing bias in estimation of $P_{m}$ and of location parameters of the two underlying distributions. When all variance components were identical across mixture components, the mixture model analyses returned parameter estimates essentially without bias and with a high degree of precision. Including random effects in the model increased the probability of correct classification substantially. No sizable differences in probability of correct classification were found between models in which a single cow effect (ig-
\end{abstract}

Received May 14, 2003.

Accepted June 30, 2003.

Corresponding author: J. Ødegård; e-mail: jorgen.odegard@ihf. nlh.no. noring relationships) was fitted and models where this effect was split into genetic and permanent environmental components, utilizing relationship information. When genetic and permanent environmental effects were fitted, the between-replicate variance of estimates of posterior means was smaller because the model accounted for random genetic drift.

(Key words: Bayesian method, mixture model, somatic cell score, udder health)

Abbreviation key: $\mathbf{C M}=$ clinical mastitis, $\mathbf{I M I}-=$ intramammary infection absent, IMI+ = intramammary infection present, $\mathbf{P E}=$ permanent environment, $\mathbf{P P M}$ $=$ posterior probability of putative mastitis, $\mathbf{P M C}=$ probability of misclassification.

\section{INTRODUCTION}

Functional traits have received increasing attention in breeding programs for dairy cattle in many countries. Mastitis resistance is one of the most important of these traits, and reducing incidence of this disease through genetic selection is of great interest both for economical and animal welfare reasons. Infection with mastitis pathogens is associated with an elevated level of SCS (Mrode and Swanson, 1996). Consequently, SCS has been used as indirect criterion in selection for reduced incidence of mastitis. However, the distribution of SCS might be regarded as a mixture of observations of SCS from healthy and diseased individuals, since groupmembership is unknown. Further, mastitis can be subcategorized, depending on the type of infection (e.g., subclinical or clinical), or on the causative udder pathogens. These categories may have specific, but overlapping distributions of SCS.

Some studies have found that a low initial SCC is associated with increased susceptibility and severity of subsequent mastitis (Schukken et al., 1994; Shuster et 
al., 1996; Schukken et al., 1999; Suriyasathaporn et al., 2000). Hence, selection for a very low SCC might impair the cows' ability to respond to infection. Therefore, statistical models for indirect genetic evaluation of susceptibility to mastitis should allow for heterogeneity in the distribution of SCS between healthy and diseased cows.

Finite mixture models are potentially useful when there is some underlying group structure in the data (McLachlan and Peel, 2000), and are typically used when the group membership of the different observations is unknown. For example, different types of mixture models have been used for identification of risks in disease mapping (Militino et al., 2001), for QTL detection (Jansen, 1992; Kao and Zeng, 1997), and for genetic analyses aiming to account for preferential treatment (Kuhn et al., 1999). Using mixture models, cows can be assigned to different subpopulations (e.g., healthy or diseased) via posterior probabilities estimated from the SCS data (Detilleux and Leroy, 2000). For SCS, a mixture model in its simplest form may be used to assign observations to two components, e.g., $\mathrm{SCS}_{1}$ and $\mathrm{SCS}_{0}$, hopefully representing SCS from cows with (IMI+) and without (IMI-) IMI (mastitis), respectively. Then, identification and culling of animals at risk could be based on the posterior probability of putative mastitis, given SCS, rather than on crude SCS. Mixture models might make better use of the information contained in SCS data, so there is a need to develop such models in the context of selection for reduced incidence of mastitis.

Detilleux and Leroy (2000) described a homoscedastic two-component mixture model with genetic effects and used the EM-algorithm for inference by maximum likelihood. The objective of our study was to develop a heteroscedastic two-component mixture model including both additive genetic and permanent environmental (PE) effects, using a Bayesian approach via the Gibbs sampler for inference. More detailed descriptions of the theory of the Gibbs sampler are given by Casella and George (1992) and Sorensen and Gianola (2002). A Gibbs sampler for a mixture model without random effects is described in McLachlan and Peel (2000).

\section{MATERIALS AND METHODS}

\section{Model}

The putative health status (group) assigned to the observations is defined by an unknown $(n \times 1)$ vector $\mathbf{z}$ $=\left\{z_{\mathrm{i}}\right\}$ of binary values. If $\mathrm{z}_{\mathrm{i}}=1$, observation $i$ is regarded as pertaining to a cow with IMI+; otherwise $\left(z_{i}=0\right)$, the observation is regarded as being from an IMI- cow.

Conditionally on $\mathbf{z}$, it was assumed that the observations could be described by the linear model:

$$
\mathbf{y}=\mathbf{X}_{\mathbf{0}} \boldsymbol{\beta}_{\mathbf{0}}+\mathbf{M}_{\mathbf{z}} \mathbf{X}_{\mathbf{1}} \boldsymbol{\beta}_{\mathbf{1}}+\mathbf{Z}_{\mathrm{a}} \mathbf{a}+\mathbf{Z}_{\mathbf{p}} \mathbf{p}+\mathbf{e},
$$

where:

$\mathbf{y}=(n \times 1)$ data vector;

$\boldsymbol{\beta}_{\mathbf{0}}=\left(f_{0} \times 1\right)$ vector of fixed effects common to all cows;

$\boldsymbol{\beta}_{\mathbf{1}}=\left(f_{1} \times 1\right)$ vector of fixed effects peculiar to cows with mastitis;

$\mathbf{a}=\left(q_{a} \times 1\right)$ vector of random additive genetic effects;

$\mathbf{p}=\left(q_{p} \times 1\right)$ vector of random permanent environmental effects;

$\mathbf{M}_{\mathbf{z}}=(n \times n)$ diagonal matrix with typical element $\mathbf{z}_{\mathrm{i}}$;

$\mathbf{e}=(n \times 1)$ vector of random residuals; and

$\mathbf{X}_{0}, \mathbf{X}_{1}, \mathbf{Z}_{\mathbf{a}}$, and $\mathbf{Z}_{\mathbf{p}}$ are incidence matrices of appropriate order.

The conditional distribution of $\mathbf{y}$, given the status vector $\mathbf{z}$, the location parameters, and some scale parameters, was assumed to be:

$$
\begin{gathered}
\mathbf{y} \mid \boldsymbol{\beta}_{0}, \boldsymbol{\beta}_{1}, \mathbf{a}, \mathbf{p}, \sigma_{e 0}^{2}, \sigma_{e 1}^{2}, \mathbf{z} \sim \\
N\left[\left(\mathbf{X}_{\mathbf{0}} \boldsymbol{\beta}_{\mathbf{0}}+\mathbf{M}_{\mathbf{z}} \mathbf{X}_{1} \boldsymbol{\beta}_{1}+\mathbf{Z}_{\mathbf{a}} \mathbf{a}+\mathbf{Z}_{\mathbf{p}} \mathbf{p}\right), \mathbf{R}\right],
\end{gathered}
$$

where

$$
\mathbf{R}=\left(\mathbf{I}_{\mathbf{n}}-\mathbf{M}_{\mathbf{z}}\right) \sigma_{e 0}^{2}+\mathbf{M}_{\mathbf{z}} \sigma_{e 1}^{2} .
$$

The parameters $\sigma_{e 0}^{2}$ and $\sigma_{e 1}^{2}$ are the residual variances within IMI- and IMI+ animals, respectively.

For the additive and PE effects it was assumed that:

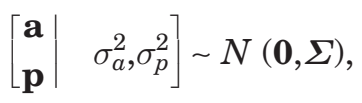

where

$$
\boldsymbol{\Sigma}=\left[\begin{array}{cc}
\mathbf{A} \sigma_{a}^{2} & \mathbf{0} \\
\mathbf{0} & \mathbf{I} \sigma_{p}^{2}
\end{array}\right] .
$$

Above, $\sigma_{a}^{2}$ is the additive genetic variance, $\sigma_{p}^{2}$ is the variance of the $\mathrm{PE}$ effects, $\mathbf{A}$ is the additive relationship matrix, and $\mathbf{I}$ is an identity matrix with dimension $q_{p}$.

\section{Bayesian Structure of the Model}

Sampling distribution of the observations given group status. By making use of the $\mathrm{n}$-dimensional multivariate normal distribution in [2], it can be shown that the density of the observation vector $\mathbf{y}$ given the status vector $\mathbf{z}$, the location parameters, and the residual variances can be written as: 


$$
\begin{gathered}
p\left(\mathbf{y} \mid \boldsymbol{\beta}_{0}, \boldsymbol{\beta}_{1}, \mathbf{a}, \mathbf{p}, \sigma_{\mathbf{e} 0}^{\mathbf{2}}, \sigma_{\mathbf{e} 1}^{\mathbf{2}}, \mathbf{z}\right) \\
=p\left(\mathbf{y} \mid \boldsymbol{\beta}_{0}, \mathbf{a}, \mathbf{p}, \sigma_{\mathbf{e} 0}^{\mathbf{2}},\left\{\mathrm{z}_{\mathrm{i}}=0\right\}\right) \times p\left(\mathbf{y} \mid \boldsymbol{\beta}_{0}, \boldsymbol{\beta}_{1}, \mathbf{a}, \mathbf{p}, \sigma_{\mathbf{e} 1}^{2},\left\{z_{\mathrm{i}}=1\right\}\right),
\end{gathered}
$$

where $\left\{z_{i}=0\right\}$ denotes the subset of the $\mathrm{n}$ observations with $z_{i}=0$ and $\left\{z^{i}=1\right\}$ denotes the subset of the $n$ observations with $z_{i}=1$. Further:

$$
\begin{gathered}
p\left(\mathbf{y} \mid \boldsymbol{\beta}_{0}, \mathbf{a}, \mathbf{p}, \sigma_{e 0}^{2},\left\{\mathbf{z}_{\mathrm{i}}=0\right\}\right) \propto \\
\left(\sigma_{e 0}^{2}\right)^{-\left(\frac{\mathrm{n}-\Sigma \mathrm{z}_{\mathrm{i}}}{2}\right)} \exp \left[-\frac{1}{2 \sigma_{e 0}^{2}}\left(\mathbf{y}-\mathbf{X}_{\mathbf{0}} \boldsymbol{\beta}_{\mathbf{0}}-\mathbf{Z}_{\mathbf{a}} \mathbf{a}-\mathbf{Z}_{\mathbf{p}} \mathbf{p}\right)^{\prime}\right. \\
\left.\left(\mathbf{I}_{\mathbf{n}}-\mathbf{M}_{\mathbf{z}}\right)\left(\mathbf{y}-\mathbf{X}_{\mathbf{0}} \boldsymbol{\beta}_{\mathbf{0}}-\mathbf{Z}_{\mathbf{a}} \mathbf{a}-\mathbf{Z}_{\mathbf{p}} \mathbf{p}\right)\right] \\
p\left(\mathbf{y} \mid \boldsymbol{\beta}_{0}, \boldsymbol{\beta}_{1}, \mathbf{a}, \mathbf{p}, \sigma_{\mathbf{e} 1}^{2},\left\{\mathbf{z}_{\mathrm{i}}=1\right\}\right) \propto \\
\left(\sigma_{e 1}^{2}\right)^{-\left(\frac{\Sigma \mathbf{z}_{\mathbf{i}}}{2}\right)} \exp _{\exp }\left[-\frac{1}{2 \sigma_{e 1}^{2}}\left(\mathbf{y}-\mathbf{X}_{\mathbf{0}} \boldsymbol{\beta}_{\mathbf{0}}-\mathbf{X}_{1} \boldsymbol{\beta}_{1}-\mathbf{Z}_{\mathbf{a}} \mathbf{a}-\mathbf{Z}_{\mathbf{p}} \mathbf{p}\right)^{\prime}\right. \\
\left.\mathbf{M}_{\mathbf{z}}\left(\mathbf{y}-\mathbf{X}_{\mathbf{0}} \boldsymbol{\beta}_{\mathbf{0}}-\mathbf{X}_{1} \boldsymbol{\beta}_{1}-\mathbf{Z}_{\mathbf{a}} \mathbf{a}-\mathbf{Z}_{\mathbf{p}} \mathbf{p}\right)\right]
\end{gathered}
$$

Prior distributions of location parameters and of the unknown status vector. A proper uniform prior distribution was assumed for the vector of fixed effects $\boldsymbol{\beta}=\left[\begin{array}{ll}\boldsymbol{\beta}_{\mathbf{0}}^{\prime} & \boldsymbol{\beta}_{\mathbf{1}}^{\prime}\end{array}\right]^{\prime}$, with density:

$$
p(\boldsymbol{\beta})=U\left[d_{1}, d_{2}\right] .
$$

To achieve a reasonably vague prior, the absolute values of the bounds $d_{1}$ and $d_{2}$ must be sufficiently large. Further, to avoid label-switching problems (McLachlan and Peel, 2000), constraints must be imposed on the parameters of the distributions of IMI- and IMI+ animals; for example, the mean SCS in the IMI- group being lower than that of the IMI+ group.

From [3] the prior densities of additive and PE effects, conditionally on the genetic and $\mathrm{PE}$ variance components, are:

$$
\begin{gathered}
p\left(\mathbf{a} \mid \sigma_{a}^{2}\right) \propto\left(\sigma_{\mathrm{a}}^{2}\right)^{-q_{a} / 2} \exp \left(-\frac{1}{2 \sigma_{a}^{2}} \mathbf{a}^{\prime} \mathbf{A}^{-\mathbf{1}} \mathbf{a}\right) \\
p\left(\mathbf{p} \mid \sigma_{p}^{2}\right) \propto\left(\sigma_{p}^{2}\right)^{-q_{p} / 2} \exp \left(-\frac{1}{2 \sigma_{p}^{2}} \mathbf{p}^{\prime} \mathbf{p}\right) .
\end{gathered}
$$

Individual elements $\left(\mathrm{z}_{\mathrm{i}}\right)$ of the classification vector $\mathbf{z}$ were assumed to be independent a priori, and following the same Bernoulli distribution:

$p\left(\mathrm{z}_{\mathrm{i}} \mid P_{m}\right)=\operatorname{Br}\left(P_{m}\right)=P_{m}^{\mathrm{z}_{\mathrm{i}}\left(1-P_{m}\right)^{1-\mathrm{z}_{\mathrm{i}}}} \quad, i=1,2, \ldots, \mathrm{n}$, where $P_{m}$ is the mixing proportion (proportion of IMI+). Hence, the corresponding multivariate distribution is:

$$
p\left(\mathbf{z} \mid P_{m}\right)=\prod_{i=1}^{n} p\left(\mathrm{z}_{\mathrm{i}}\right)=P_{m}^{\Sigma \mathrm{z}_{\mathrm{i}}}\left(1-P_{m}\right)^{n-\Sigma \mathrm{z}_{\mathrm{i}}} .
$$

Note that if the expected value of [1] is taken with respect to the prior distributions of $\mathbf{z}, \mathbf{a}$, and $\mathbf{p}$ (but conditionally on $\boldsymbol{\beta}_{0}$ and $\boldsymbol{\beta}_{1}$ ) one obtains:

$$
E\left(\mathbf{y} \mid \boldsymbol{\beta}_{\mathbf{0}}, \boldsymbol{\beta}_{1}\right)=\left(1-P_{m}\right) \mathbf{X}_{\mathbf{0}} \boldsymbol{\beta}_{\mathbf{0}}+P_{m}\left(\mathbf{X}_{\mathbf{0}} \boldsymbol{\beta}_{\mathbf{0}}+\mathbf{X}_{\mathbf{1}} \boldsymbol{\beta}_{\mathbf{1}}\right) .
$$

This is the usual representation for the mean vector of a two-component mixture from a distribution with mean vector $\mathbf{X}_{\mathbf{0}} \boldsymbol{\beta}_{\mathbf{0}}$ (and proportion 1- $P_{m}$ ) and another distribution with mean vector $\mathbf{X}_{\mathbf{0}} \boldsymbol{\beta}_{\mathbf{0}}+\mathbf{X}_{\mathbf{1}} \boldsymbol{\beta}_{\mathbf{1}}$ (and proportion $P_{m}$ ).

Priors for variance components and mixing proportion. Independent conjugate priors were used for the four variance components and for the mixing proportion. The dispersion parameters were assigned scale inverted $\chi$-square prior distributions, with densities:

$$
\begin{gathered}
p\left(\sigma_{a}^{2}\right) \propto\left(\sigma_{a}^{2}\right)^{-\left(\nu_{a}+2\right) / 2} \exp \left(-\frac{1}{2} \nu_{a} s_{a}^{2} / \sigma_{a}^{2}\right) \\
p\left(\sigma_{p}^{2}\right) \propto\left(\sigma_{p}^{2}\right)^{-\left(\nu_{p}+2\right) / 2} \exp \left(-\frac{1}{2} \nu_{p} s_{p}^{2} / \sigma_{p}^{2}\right) ; \\
p\left(\sigma_{e 0}^{2}\right) \propto\left(\sigma_{e 0}^{2}\right)^{-\left(\nu_{e 0}+2\right) / 2} \exp \left(-\frac{1}{2} \nu_{e 0} s_{e 0}^{2} / \sigma_{e 0}^{2}\right) ; \text { and } \\
p\left(\sigma_{e 1}^{2}\right) \propto\left(\sigma_{e 1}^{2}\right)^{-\left(\nu_{e 1}+2\right) / 2} \exp \left(-\frac{1}{2} \nu_{e 1} s_{e 1}^{2} / \sigma_{e 1}^{2}\right),
\end{gathered}
$$

where the $\nu$ and $s^{2}$ are known hyper-parameters. In this study, all $\nu$ were set equal to 2 , while the $s^{2}$ equaled the true variance component values used in the simulations.

The mixing proportion $\left(P_{m}\right)$, taking values between zero and one, was assigned a beta prior distribution with hyper-parameters $\alpha_{1}$, and $\alpha_{2}$. In this study, both parameters were set to 2 . The density is:

$$
p\left(P_{m}\right)=\operatorname{Be}\left(\alpha_{1}, \alpha_{2}\right) \propto P_{m}{ }^{\alpha_{1}-1}\left(1-P_{m}\right)^{\alpha_{2}-1} .
$$

Joint posterior distribution. The joint density of $\mathbf{y}, \boldsymbol{\beta}, \mathbf{p}, \mathbf{a}$, and $\mathbf{z}$, conditional on the dispersion parameters and mixing proportion, is:

$$
\begin{gathered}
p\left(\mathbf{y}, \boldsymbol{\beta}, \mathbf{a}, \mathbf{p}, \mathbf{z} \mid \sigma_{a}^{2}, \sigma_{p}^{2}, \sigma_{e 0}^{2}, \sigma_{e 1}^{2}, P_{m}\right) \\
\propto[4] \times[5] \times[6] \times[7] .
\end{gathered}
$$

The joint posterior density of all unknown parameters is given by: 


$$
\begin{aligned}
& p\left(\boldsymbol{\beta}, \mathbf{a}, \mathbf{p}, \mathbf{z}, \sigma_{a}^{2}, \sigma_{p}^{2}, \sigma_{e 0}^{2}, \sigma_{e 1}^{2}, P_{m} \mid \mathbf{y}\right) \\
\propto & p\left(\mathbf{y}, \boldsymbol{\beta}, \mathbf{a}, \mathbf{p}, \mathbf{z} \mid \sigma_{a}^{2}, \sigma_{p}^{2}, \sigma_{e 0}^{2}, \sigma_{e 1}^{2}, P_{m}\right) \\
& p\left(\sigma_{a}^{2}\right) p\left(\sigma_{p}^{2}\right) p\left(\sigma_{e 0}^{2}\right) p\left(\sigma_{e 1}^{2}\right) p\left(P_{m}\right)
\end{aligned}
$$

Explicitly:

$$
\begin{aligned}
& \left(\sigma_{e 0}^{2}\right)^{-\left(\frac{n-\Sigma z_{i}+\nu_{e 0}+2}{2}\right)} \\
& \times \exp \left[-\frac{1}{2 \sigma_{e 0}^{2}}\left\{\left(\mathbf{y}-\mathbf{X}_{\mathbf{0}} \boldsymbol{\beta}_{0}-\mathbf{Z}_{\mathbf{a}} \mathbf{a}-\mathbf{Z}_{\mathbf{p}} \mathbf{p}\right)^{\prime}\right.\right. \\
& \left.\left.\left(\mathbf{I}_{\mathbf{n}}-\mathbf{M}_{\mathbf{z}}\right)\left(\mathbf{y}-\mathbf{X}_{\mathbf{0}} \boldsymbol{\beta}_{\mathbf{0}}-\mathbf{Z}_{\mathbf{a}} \mathbf{a}-\mathbf{Z}_{\mathbf{p}} \mathbf{p}\right)+\nu_{e 0} s_{e o}^{2}\right\}\right] \\
& \times\left(\sigma_{e 1}^{2}\right)^{-\left(\frac{\Sigma z_{i}+\nu_{e 1}+2}{2}\right)} \\
& \times \exp \left[-\frac{1}{2 \sigma_{e 1}^{2}}\left\{\left(\mathbf{y}-\mathbf{X}_{\mathbf{0}} \boldsymbol{\beta}_{\mathbf{0}}-\mathbf{X}_{1} \boldsymbol{\beta}_{1}-\mathbf{Z}_{\mathbf{a}} \mathbf{a}-\mathbf{Z}_{\mathbf{p}} \mathbf{p}\right)^{\prime}\right.\right. \\
& \left.\left.\mathbf{M}_{\mathbf{z}}\left(\mathbf{y}-\mathbf{X}_{\mathbf{0}} \boldsymbol{\beta}_{\mathbf{0}}-\mathbf{X}_{1} \boldsymbol{\beta}_{1}-\mathbf{Z}_{\mathbf{a}} \mathbf{a}-\mathbf{Z}_{\mathbf{p}} \mathbf{p}\right)+\nu_{e 1} s_{e 1}^{2}\right\}\right] \\
& \times\left(\sigma_{a}^{2}\right)^{-\left(\frac{q_{a}+\nu_{a}+2}{2}\right)} \exp \left[-\frac{1}{2 \sigma_{a}^{2}}\left(\mathbf{a}^{\prime} \mathbf{A}^{-1} \mathbf{a}+\nu_{\mathbf{a}} s_{a}^{2}\right)\right] \\
& \times\left(\sigma_{p}^{2}\right)^{-\left(\frac{q_{p}+\nu_{p+2}}{2}\right)} \exp \left[-\frac{1}{2 \sigma_{p}^{2}}\left(\mathbf{p}^{\prime} \mathbf{p}+\nu_{p} s_{p}^{2}\right)\right] \\
& \times P_{m}^{\sum z_{i}+\alpha 1^{-1}}\left(1-P_{m}\right)^{n-\Sigma z_{i}+\alpha_{2}-1} .
\end{aligned}
$$

Fully conditional distributions. The conditional posterior distributions of each parameter (or block of parameters) are required for implementing a Gibbs sampler. Let:

$$
\mathbf{W}=\left[\begin{array}{llll}
\mathbf{X}_{\mathbf{0}} & \mathbf{M}_{\mathbf{z}} \mathbf{X}_{1} & \mathbf{Z}_{\mathbf{a}} & \mathbf{Z}_{\mathbf{p}}
\end{array}\right]
$$

and let the location parameters be represented by:

$$
\theta=\left[\begin{array}{llll}
\beta_{0}^{\prime} & \beta_{1}^{\prime} & \mathbf{a}^{\prime} & \mathbf{p}^{\prime}
\end{array}\right]^{\prime} .
$$

Hence, one can write [1] as:

$$
\mathbf{y}=\mathbf{W} \boldsymbol{\theta}+\mathbf{e},
$$

and let $\Omega=\left[\begin{array}{cc}\mathbf{0} & \mathbf{0} \\ \mathbf{0} & \boldsymbol{\Sigma}^{-1}\end{array}\right]$, with $\boldsymbol{\Sigma}$ defined as in [3].
The fully conditional posterior distribution of the parameter vector $\boldsymbol{\theta}$ (e.g., Sorensen and Gianola, 2002) is:

$$
\boldsymbol{\theta} \mid \sigma_{a}^{2}, \sigma_{p}^{2}, \sigma_{e 0}^{2}, \sigma_{e 1}^{2}, P_{m}, \mathbf{z}, \mathbf{y} \sim N\left(\hat{\boldsymbol{\theta}}, \mathbf{C}^{-1}\right),
$$

where $\hat{\boldsymbol{\theta}}=\mathbf{C}^{-1} \mathbf{W}^{\prime} \mathbf{R}^{-\mathbf{1}} \mathbf{y}=\mathbf{C}^{-\mathbf{1}} \mathbf{r}, \mathbf{C}=\left[\mathbf{W}^{\prime} \mathbf{R}^{-1} \mathbf{W}+\Omega\right]$, and $\mathbf{R}=\left[\left(\mathbf{I}_{\mathbf{n}}-\mathbf{M}_{\mathbf{z}}\right) \sigma_{e 0}^{2}+\mathbf{M}_{\mathbf{z}} \sigma_{e 1}^{2}\right]$, as defined earlier.

Alternatively, one can select elements of interest in $\theta$, denote them as $\theta_{1}$, and then reorder $\theta$ as:

$$
\theta=\left[\begin{array}{l}
\theta_{1} \\
\theta_{2}
\end{array}\right]
$$

Let $\mathbf{r}$ and $\mathbf{C}$ be partitioned accordingly. Define

$$
\tilde{\boldsymbol{\theta}}_{\mathbf{1}}=\boldsymbol{E}\left(\boldsymbol{\theta}_{\mathbf{1}} \mid \boldsymbol{\theta}_{\mathbf{2}}, \sigma_{a}^{2}, \sigma_{p}^{2}, \sigma_{e 0}^{2}, \sigma_{e 1}^{2}, P_{m}, \mathbf{z}, \mathbf{y}\right)=\mathbf{C}_{\mathbf{1 1}}^{-1}\left[\mathbf{r}_{\mathbf{1}}-\mathbf{C}_{\mathbf{1 2}} \boldsymbol{\theta}_{\mathbf{2}}\right]
$$

The fully conditional posterior distribution of any partition of the parameter vector $\theta$, given all other parameters in the vector (Sorensen and Gianola, 2002), is:

$$
\boldsymbol{\theta}_{\mathbf{1}} \mid \boldsymbol{\theta}_{\mathbf{2}}, \sigma_{a}^{2}, \sigma_{p}^{2}, \sigma_{e 0}^{2}, \sigma_{e 1}^{2}, P_{m}, \mathbf{z}, \mathbf{y} \sim N\left(\tilde{\boldsymbol{\theta}}_{\mathbf{1}}, \mathbf{C}_{\mathbf{1 1}}{ }^{-\mathbf{1}}\right)
$$

The fully conditional posterior density of the variance components can be deduced from [9]. The fully conditional posterior density of the genetic variance is:

$$
\begin{gathered}
p\left(\sigma_{a}^{2} \mid \boldsymbol{\theta}, \sigma_{p}^{2}, \sigma_{e 0}^{2}, \sigma_{e 1}^{2}, \sigma_{e 1}^{2}, P_{m}, \mathbf{y}, \mathbf{z}\right) \\
\propto\left(\sigma_{a}^{2}\right)^{-\left(\frac{q_{a}+\nu_{a}+2}{2}\right)} \times \exp \left[-\frac{1}{2 \sigma_{a}^{2}}\left(\mathbf{a}^{\prime} \mathbf{A}^{-1} \mathbf{a}+\nu_{a} s_{a}^{2}\right)\right],
\end{gathered}
$$

which is in the form of a scaled inverted $\chi$-square density, with $\left[q_{a}+\nu_{a}\right]$ degrees of freedom and scale parameter $\left[\mathbf{a}^{\prime} \mathbf{A}^{-1} \mathbf{a}+\nu_{a} s_{a}^{2}\right]$.

Likewise, the fully conditional posterior density of the $\mathrm{PE}$ variance is:

$$
\begin{aligned}
& p\left(\sigma_{p}^{2} \mid \boldsymbol{\theta}, \sigma_{a}^{2}, \sigma_{e 0}^{2}, \sigma_{e 1}^{2}, P_{m}, \mathbf{y}, \mathbf{z}\right) \\
& \propto\left(\sigma_{p}^{2}\right)^{-\left(\frac{q_{p}+\nu_{p}+2}{2}\right)} \times \exp \left[-\frac{1}{2 \sigma_{p}^{2}}\left(\mathbf{p}^{\prime} \mathbf{p}+\nu_{p} s_{p}^{2}\right)\right],
\end{aligned}
$$

which is in the form of a scaled inverted $\chi$-square density, with $\left[q_{p}+\nu_{p}\right]$ degrees of freedom and scale parameter $\left[\mathbf{p}^{\prime} \mathbf{p}+\nu_{p} s_{p}^{2}\right]$.

From [9] the fully conditional density of the residual variance for IMI- observations is:

Journal of Dairy Science Vol. 86, No. 11, 2003 


$$
\begin{aligned}
& p\left(\sigma_{e 0}^{2} \mid \boldsymbol{\theta}, \sigma_{a}^{2}, \sigma_{p}^{2}, \sigma_{e 1}^{2}, P_{m}, \mathbf{y}, \mathbf{z}\right) \\
& \propto\left(\sigma_{e 0}^{2}\right)^{-\left(\frac{n^{-\Sigma z_{i}+\nu_{e 0}+2}}{2}\right)} \\
& \times \exp \left[-\frac{1}{2 \sigma_{e 0}^{2}}\left\{\left(\mathbf{y}-\mathbf{X}_{\mathbf{0}} \boldsymbol{\beta}_{\mathbf{0}}-\mathbf{Z}_{\mathbf{a}} \mathbf{a}-\mathbf{Z}_{\mathbf{p}} \mathbf{p}\right)^{\prime}\right.\right. \\
& \left.\left.\left(\mathbf{I}_{\mathbf{n}}-\mathbf{M}_{\mathbf{z}}\right)\left(\mathbf{y}-\mathbf{X}_{\mathbf{0}} \boldsymbol{\beta}_{\mathbf{0}}-\mathbf{Z}_{\mathbf{a}} \mathbf{a}-\mathbf{Z}_{\mathbf{p}} \mathbf{p}\right)+\nu_{e 0} s_{e 0}^{2}\right\}\right],
\end{aligned}
$$

which is in the form of a scaled inverted $\chi$-square density, with $\left[n-\Sigma z_{i}+\nu_{e 0}\right]$ degrees of freedom, and with scale parameter:

$$
\begin{gathered}
{\left[\left(\mathbf{y}-\mathbf{X}_{\mathbf{0}} \boldsymbol{\beta}_{\mathbf{0}}-\mathbf{Z}_{\mathbf{a}} \mathbf{a}-\mathbf{Z}_{\mathbf{p}} \mathbf{p}\right)^{\prime}\right.} \\
\left.\left(\mathbf{I}_{\mathbf{n}}-\mathbf{M}_{\mathbf{z}}\right)\left(\mathbf{y}-\mathbf{X}_{\mathbf{0}} \boldsymbol{\beta}_{\mathbf{0}}-\mathbf{Z}_{\mathbf{a}} \mathbf{a}-\mathbf{Z}_{\mathbf{p}} \mathbf{p}\right)+\nu_{e 0} s_{e 0}^{2}\right] .
\end{gathered}
$$

The corresponding fully conditional posterior density of the residual variance for IMI+ observations is:

$$
\begin{gathered}
p\left(\sigma_{e 1}^{2} \mid \boldsymbol{\theta}, \sigma_{a}^{2}, \sigma_{p}^{2}, \sigma_{e 0}^{2}, \mathbf{y}, \mathbf{z}\right) \\
\propto\left(\sigma_{e 1}^{2}\right)^{-\left(\frac{\Sigma z_{i}^{+\nu}{ }_{e 1}+2}{2}\right)} \\
\times \exp \left[-\frac{1}{2 \sigma_{e 1}^{2}}\left\{\left(\mathbf{y}-\mathbf{X}_{\mathbf{0}} \boldsymbol{\beta}_{\mathbf{0}}-\mathbf{X}_{\mathbf{1}} \boldsymbol{\beta}_{\mathbf{1}}-\mathbf{Z}_{\mathbf{a}} \mathbf{a}-\mathbf{Z}_{\mathbf{p}} \mathbf{p}\right)^{\prime}\right.\right. \\
\left.\left.\mathbf{M}_{\mathbf{z}}\left(\mathbf{y}-\mathbf{X}_{\mathbf{0}} \boldsymbol{\beta}_{\mathbf{0}}-\mathbf{X}_{\mathbf{1}} \boldsymbol{\beta}_{\mathbf{1}}-\mathbf{Z}_{\mathbf{a}} \mathbf{a}-\mathbf{Z}_{\mathbf{p}} \mathbf{p}\right)+\nu_{e 1} s_{e 1}^{2}\right\}\right],
\end{gathered}
$$

which is in the form of a scaled inverted $\chi$-square density, with $\left[\Sigma z_{i}+\nu_{e 1}\right]$ degrees of freedom, and with scale parameter:

$$
\begin{gathered}
{\left[\left(\mathbf{y}-\mathbf{X}_{\mathbf{0}} \boldsymbol{\beta}_{\mathbf{0}}-\mathbf{X}_{\mathbf{1}} \boldsymbol{\beta}_{\mathbf{1}}-\mathbf{Z}_{\mathbf{a}} \mathbf{a}-\mathbf{Z}_{\mathbf{p}} \mathbf{p}\right)^{\prime}\right.} \\
\left.\mathbf{M}_{\mathbf{z}}\left(\mathbf{y}-\mathbf{X}_{\mathbf{0}} \boldsymbol{\beta}_{\mathbf{0}}-\mathbf{X}_{\mathbf{1}} \boldsymbol{\beta}_{\mathbf{1}}-\mathbf{Z}_{\mathbf{a}} \mathbf{a}-\mathbf{Z}_{\mathbf{p}} \mathbf{p}\right)+\nu_{e 1} s_{e 1}^{2}\right] .
\end{gathered}
$$

The fully conditional posterior distribution of an individual $\mathrm{z}_{\mathrm{i}}$ is Bernoulli, and from [9] it can be shown that the $z_{i}$ are mutually independent, given all other parameters. The parameter $(\tau(\cdot))$ of the fully conditional Bernoulli distribution of $\mathrm{z}_{\mathrm{i}}$ (McLachlan and Peel, 2000) is:

$$
\begin{gathered}
\tau\left(\mathrm{z}_{\mathrm{i}} \mid \boldsymbol{\theta}, \sigma_{a}^{2}, \sigma_{p}^{2}, \sigma_{e 0}^{2}, \sigma_{e 1}^{2}, P_{m}, \mathrm{y}_{\mathrm{i}}\right)= \\
\operatorname{Pr}\left(\mathrm{z}_{\mathrm{i}}=1 \mid \boldsymbol{\theta}, \sigma_{a}^{2}, \sigma_{p}^{2}, \sigma_{e 0}^{2}, \sigma_{e 1}^{2}, P_{m}, \mathrm{y}_{\mathrm{i}}\right) \\
=\frac{p\left(\mathrm{y}_{\mathrm{i}} \mid \boldsymbol{\theta}, \sigma_{e 1}^{2}, \mathrm{z}_{\mathrm{i}}=1\right) P_{m}}{p\left(\mathrm{y}_{\mathrm{i}} \mid \boldsymbol{\theta}, \sigma_{e 0}^{2}, \mathrm{z}_{\mathrm{i}}=0\right)\left(1-P_{m}\right)+p\left(\mathrm{y}_{\mathrm{i}} \mid \boldsymbol{\theta}, \sigma_{e 1}^{2}, \mathrm{z}_{\mathrm{i}}=1\right) P_{m}},
\end{gathered}
$$

where it can be shown from [9] that:

$$
\begin{gathered}
p\left(\mathrm{y}_{\mathrm{i}} \mid \boldsymbol{\theta}, \sigma_{e 0}^{2}, \mathrm{z}_{\mathrm{i}}=0\right)=\left(2 \pi \sigma_{e 0}^{2}\right)^{-\frac{1}{2}} \\
\exp \left[-\frac{1}{2 \sigma_{e 0}^{2}}\left(\mathrm{y}_{\mathrm{i}}-\mathbf{x}_{\mathbf{o i}}^{\prime} \boldsymbol{\beta}_{\mathbf{0}}-\mathbf{z}_{\mathbf{a i}}^{\prime} \mathbf{a}-\mathbf{z}_{\mathbf{p i}}^{\prime} \mathbf{p}\right)^{2}\right], \text { and } \\
p\left(\mathrm{y}_{\mathrm{i}} \mid \boldsymbol{\theta}, \sigma_{e 1}^{2}, \mathrm{z}_{\mathrm{i}}=1\right)=\left(2 \pi \sigma_{e 1}^{2}\right)^{-\frac{1}{2}} \\
\exp \left[-\frac{1}{2 \sigma_{e 1}^{2}}\left(\mathrm{y}_{\mathrm{i}}-\mathbf{x}_{\mathbf{o i}}^{\prime} \boldsymbol{\beta}_{\mathbf{0}}-\mathbf{x}_{\mathbf{1 i}}^{\prime} \boldsymbol{\beta}_{\mathbf{1}}-\mathbf{z}_{\mathbf{a i}}^{\prime} \mathbf{a}-\mathbf{z}_{\mathbf{p i}}^{\prime} \mathbf{p}\right)^{2}\right]
\end{gathered}
$$

with $\mathbf{x}_{\mathbf{o i}}^{\prime}, \mathbf{x}_{\mathbf{1 i}}^{\prime}, \mathbf{z}_{\mathbf{a i}}^{\prime}$, and $\mathbf{z}_{\mathbf{p i}}^{\prime}$ being the $i$ th rows of $\mathbf{X}_{\mathbf{o}}, \mathbf{x}_{\mathbf{1}}$, $\mathbf{Z}_{\mathbf{a}}$, and $\mathbf{Z}_{\mathbf{p}}$, respectively.

Finally, the fully conditional posterior density of $\mathrm{Pm}$ is deduced from [9] to be:

$$
\begin{gathered}
p\left(P_{m} \mid \boldsymbol{\theta}, \sigma_{a}^{2}, \sigma_{p}^{2}, \sigma_{e 0}^{2}, \sigma_{e 1}^{2}, \mathbf{y}, \mathbf{z}\right) \\
\propto P_{m}{ }^{\sum \mathrm{z}_{\mathrm{i}}+\alpha_{1}-1}\left(1-P_{m}\right)^{n-\Sigma \mathrm{z}_{\mathrm{i}}+\alpha_{2}-1}
\end{gathered}
$$

which is in the form of a beta-distribution with parameters $\left(\Sigma \mathrm{z}_{\mathrm{i}}+\alpha_{1}\right.$ and $\left(n-\Sigma \mathrm{z}_{\mathrm{i}}+\alpha_{2}\right)$.

\section{Implementation of a Gibbs Sampler}

The following steps describe how Gibbs sampling can be implemented for our two-component mixture model:

1) Set initial values for parameters as needed.

2) Select a block $\left(\theta_{1}\right)$ of the vector $\theta$, and compute;

$$
\tilde{\theta}_{1}=\mathbf{C}_{11}^{-1}\left[\mathbf{r}_{1}-\mathbf{C}_{12} \theta_{2}\right],
$$

where $\theta_{2}$ includes all elements in $\theta$, except those contained in $\theta_{1}$. Sample a new $\boldsymbol{\theta}_{1}$ from $N\left(\tilde{\boldsymbol{\theta}}_{\mathbf{1}}, \mathbf{C}_{\mathbf{1 1}}^{-\mathbf{1}}\right)$. Continue sampling new blocks from $\theta$ until all elements are updated.

3) Compute $\mathbf{a}^{\prime} \mathbf{A}^{-\mathbf{1}} \mathbf{a}$, and replace $\sigma_{a}^{2}$ with $\left(\mathbf{a}^{\prime} \mathbf{A}^{-\mathbf{1}} \mathbf{a}+\right.$ $\left.\nu_{a} s_{a}^{2}\right) / \chi_{q_{a}+\nu_{a}}^{2}$, where $\chi_{q_{a}+\nu_{a}}^{2}$ is a random draw from a central $\chi$-square distribution with $\left[\nu_{a}+q_{a}\right]$ degrees of freedom.

4) Compute $\mathbf{p}^{\prime} \mathbf{p}$, and replace $\sigma_{p}^{2}$ by $\left(\mathbf{p}^{\prime} \mathbf{p}+\nu_{p} s_{p}^{2}\right) / \chi_{q_{p}+\nu_{p}}^{2}$, where $\chi_{q_{p}+\nu_{p}}^{2}$ is a random draw from a central $\chi$ square distribution with $\left[\nu_{p}+q_{p}\right]$ degrees of freedom.

5) Compute $(\mathbf{y}-\mathbf{W} \boldsymbol{\theta})^{\prime}\left(\mathbf{I}_{\mathbf{n}}-\mathbf{M}_{\mathbf{z}}\right)(\mathbf{y}-\mathbf{W} \boldsymbol{\theta})$ and replace $\sigma_{e 0}^{2}$ with

$$
\left[(\mathbf{y}-\mathbf{W} \boldsymbol{\theta})^{\prime}\left(\mathbf{I}_{\mathbf{n}}-\mathbf{M}_{\mathbf{z}}\right)(\mathbf{y}-\mathbf{W} \boldsymbol{\theta})+\nu_{e 0} s_{e 0}^{2}\right] / \chi_{n-\Sigma \mathbf{z}_{\mathrm{i}}}^{2}+\nu_{e 0},
$$

where $\chi_{n-\Sigma \mathrm{z}_{\mathrm{i}}}^{2}+\nu_{e 0}$ is a random draw from a central $\chi$-square distribution with $\left[n-\Sigma \mathrm{z}_{\mathrm{i}}+\nu_{e 0}\right]$ degrees of freedom.

6) Compute $(\mathbf{y}-\mathbf{W} \boldsymbol{\theta})^{\prime} \mathbf{M}_{\mathbf{z}}(\mathbf{y}-\mathbf{W} \boldsymbol{\theta})$ and replace $\sigma_{e 1}^{2}$ by 


$$
\left[(\mathbf{y}-\mathbf{W} \boldsymbol{\theta})^{\prime} \mathbf{M}_{\mathbf{z}}(\mathbf{y}-\mathbf{W} \boldsymbol{\theta})+\nu_{e 1} s_{e 1}^{2}\right] / \chi_{\Sigma \mathrm{z}_{\mathrm{i}}}^{2}+\nu_{e 0}
$$

where $\chi_{\Sigma \mathrm{z}_{\mathrm{i}}}^{2}+\nu_{e 0}$ is a random draw from a central $\chi$ square distribution with $\left[\Sigma z_{i}+\nu_{e 1}\right]$ degrees of freedom.

7) Compute $\tau(\cdot)$ as in [15] and sample $z_{i}$ from a Bernoulli distribution with parameter $\tau(\cdot)$ for $i=1,2$, $\ldots, n$.

8) Sample $P_{m}$ from a beta distribution with parameters $\left(\Sigma \mathrm{z}_{\mathrm{i}}+\alpha_{1}\right)$ and $\left(n-\Sigma \mathrm{z}_{\mathrm{i}}+\alpha_{2}\right)$.

9) Repeat (2 through 8) $k$ times for burn-in as needed. Then, sample $\mathbf{z}, \boldsymbol{\theta}, \sigma_{a}^{2}, \sigma_{p}^{2}, \sigma_{e 0}^{2}, \sigma_{e 1}^{2}$, and $P_{m} m$ times. The total number of iterations is thus $k+m$. The values of $\mathrm{k}$ and $\mathrm{m}$ can be chosen based on visual inspection of trace plots and on convergence diagnostics (Raftery and Lewis, 1992).

\section{Simulation Studies}

The mixture model was evaluated using simulated SCS drawn from a two-component normal mixture distribution. When included, genetic and PE effects were considered as having the same distributions across mixture components.

Simulation I. A total of 8000 SCS observations was simulated for animals with IMI- $\left(\mathrm{SCS}_{0}\right)$ and IMI+ $\left(\mathrm{SCS}_{1}\right)$ statuses, as follows:

$$
\begin{gathered}
\mathbf{S C S}_{\mathbf{0}}=\mathbf{1} \mu_{0}+\mathbf{e}_{\mathbf{0}}, \text { and } \\
\mathbf{S C S}_{\mathbf{1}}=\mathbf{1} \mu_{1}+\mathbf{e}_{\mathbf{1}},
\end{gathered}
$$

where $\mu_{0}$ and $\mu_{1}$ are the means of the two distributions, and

$$
\mathbf{e}_{\mathbf{0}} \sim N\left(\mathbf{0}, \mathbf{I} \sigma_{\mathbf{e} 0}^{2}\right), \text { and } \mathbf{e}_{\mathbf{1}} \sim N\left(\mathbf{0}, \mathbf{I} \sigma_{e 1}^{2}\right) .
$$

Cows were assigned to the two groups at random using appropriate membership probabilities $\left(P_{m}\right)$. If observation $i$ was assigned to the IMI+ group, $\mathrm{SCS}_{\mathrm{i}}$ equaled $\mathrm{SCS}_{1 \mathrm{i}}$; otherwise $\mathrm{SCS}_{\mathrm{i}}$ was equal to $\mathrm{SCS}_{0 \mathrm{i}}$. The trait SCS was, therefore, a mixture of observations drawn from $\mathbf{S C S}_{\mathbf{0}}$ and $\mathbf{S C S}_{\mathbf{1}}$, with a distance $\Delta=\left(\mu_{1}-\mu_{0}\right)$ between the means of the two distributions. Parameters for nine different simulated settings are presented in Table 1. Each setting was replicated five times, with this number dictated by computing considerations. Settings 1 to 4 allowed for different degrees of heterogeneous residual variance but had the same $\mu_{0}$ (4.0), $\mu_{1}$ (7.0), and $P_{m}(0.25)$ values. Settings 5 to 9 were homoscedastic $\left(\sigma_{e 0}^{2}=\sigma_{e 1}^{2}=1.0\right)$, but had varying levels of $\mu_{0}$, $\mu_{1}$, and $P_{m}$.

The following mixture model (given $\mathbf{z}$ ) was used to analyze the simulated datasets:
$\mathbf{S C S}=\mathbf{1} \mu_{0}+\mathbf{z} \Delta+\mathbf{e}$,

where

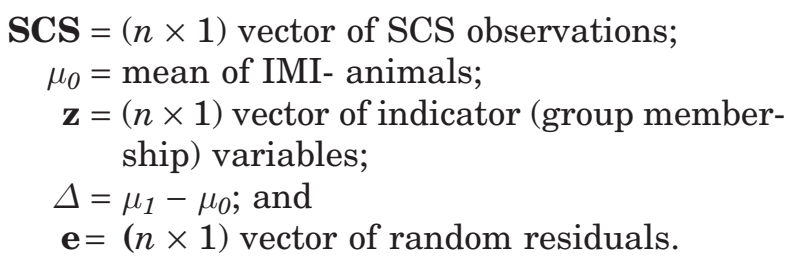

In the Gibbs sampler, values of $k=1000$ and $m=$ 30,000 were used. To avoid label-switching problems, only samples where $\Delta>0$ were accepted.

Simulation II. A total of 32,000 SCS observations were simulated, for four discrete generations, with 800 cows and 10 bulls per generation, and each cow having 10 observations (e.g., test-day records). No selection was applied, sires were recruited from 10 different bull dams, and all sires in each generation had the same probability of siring offspring of both sexes. Each cow was replaced by a daughter, and mating was at random. Breeding values for base animals were sampled from a normal distribution with mean 0 , and variance $\sigma_{a}^{2}$, while breeding values for nonbase animals were sampled from a normal distribution with the midparent value as mean, and variance $0.5 \sigma_{a}^{2}$. Inbreeding was ignored.

Somatic cell scores from healthy $\left(\mathrm{SCS}_{0}\right)$ and infected $\left(\mathrm{SCS}_{1}\right)$ animals were simulated as follows:

$$
\begin{gathered}
\mathbf{S C S}_{\mathbf{0}}=\mathbf{1} \mu_{0}+\mathbf{a}+\mathbf{p}+\mathbf{e}_{\mathbf{0}}, \text { and } \\
\mathbf{S C S}_{\mathbf{1}}=\mathbf{1} \mu_{1}+\mathbf{a}+\mathbf{p}+\mathbf{e}_{\mathbf{1}}
\end{gathered}
$$

where $\mu_{0}$ and $\mu_{1}$ are the means of the two distributions,

$$
\begin{gathered}
\mathbf{a} \sim N\left(\mathbf{0}, \mathbf{A} \sigma_{a}^{2}\right), \mathbf{p} \sim N\left(\mathbf{0}, \mathbf{I} \sigma_{p}^{2}\right), \\
\mathbf{e}_{\mathbf{0}} \sim N\left(\mathbf{0}, \mathbf{I} \sigma_{e 0}^{2}\right), \text { and } \mathbf{e}_{\mathbf{1}} \sim N\left(\mathbf{0}, \mathbf{I} \sigma_{e 1}^{2}\right) .
\end{gathered}
$$

As before, cows were assigned to the two status groups at random, using the appropriate $P_{m}$ values. Parameters for the two settings are given in Table 2. Both settings had five replicates.

The simulated datasets were analyzed with the following three statistical models:

$$
\begin{aligned}
& \text { Model 1: } \mathbf{S C S}=\mathbf{1} \mu_{0}+\mathbf{z} \Delta+\mathbf{e} \\
& \text { Model 2: } \mathbf{S C S}=\mathbf{1} \mu_{0}+\mathbf{z} \Delta+\mathbf{Z}_{\mathbf{p}} \mathbf{c}+\mathbf{e} \\
& \text { Model 3: SCS }=\mathbf{1} \mu_{0}+\mathbf{z} \Delta+\mathbf{Z}_{\mathbf{a}} \mathbf{a}+\mathbf{Z}_{\mathbf{p}} \mathbf{p}+\mathbf{e}
\end{aligned}
$$

where model 1 is equal to the model defined in simulation I, and $\mathbf{a}, \mathbf{p}, \mathbf{Z}_{\mathbf{a}}$, and $\mathbf{Z}_{\mathbf{p}}$ are as defined in [1]. The factor $\mathbf{c}$ is a $\left(q_{p} \times 1\right)$ vector of random cow effects, and 
Table 1. Input parameters (IP) for nine settings in simulation I and their posterior means (PM) from a mixture model, averaged over 5 replicates. Standard deviations (SD) of PM from the 5 replicates are placed in brackets. Parameters for simulated SCS are: mean of cows with health status IMI- $\left(\mu_{0}\right)$ and IMI+ $\left(\mu_{1}\right)$, mixing proportion $\left(P_{m}\right)$, and the respective residual variances for cows with IMI- and IMI+ $\left(\sigma_{e 0}^{2}\right.$ and $\left.\sigma_{e 1}^{2}\right)$.

\begin{tabular}{|c|c|c|c|c|c|}
\hline Setting & $\mu_{0}$ & $\mu_{1}$ & $P_{m}$ & $\sigma_{e 0}^{2}$ & $\sigma_{e 1}^{2}$ \\
\hline \multicolumn{6}{|l|}{1} \\
\hline IP & 4.0 & 7.0 & 0.25 & 0.50 & 0.75 \\
\hline PM & 4.0256 & 7.0601 & 0.2487 & 0.5214 & 0.6864 \\
\hline SD & $(0.0113)$ & $(0.0276)$ & $(0.0063)$ & $(0.0053)$ & $(0.0194)$ \\
\hline \multicolumn{6}{|l|}{2} \\
\hline IP & 4.0 & 7.0 & 0.25 & 1.00 & 1.50 \\
\hline PM & 4.0858 & 7.2540 & 0.2155 & 1.0929 & 1.1991 \\
\hline SD & $(0.0116)$ & $(0.0720)$ & $(0.0050)$ & $(0.0168)$ & $(0.0132)$ \\
\hline \multicolumn{6}{|l|}{3} \\
\hline IP & 4.0 & 7.0 & 0.25 & 2.00 & 3.00 \\
\hline PM & 4.1480 & 7.6418 & 0.1752 & 2.1838 & 2.2520 \\
\hline SD & $(0.0238)$ & $(0.0999)$ & $(0.0109)$ & $(0.0490)$ & $(0.0607)$ \\
\hline \multicolumn{6}{|l|}{4} \\
\hline IP & 4.0 & 7.0 & 0.25 & 1.50 & 1.00 \\
\hline PM & 3.8674 & 6.7307 & 0.3051 & 1.3365 & 1.2745 \\
\hline SD & $(0.0242)$ & $(0.0146)$ & $(0.0060)$ & $(0.0290)$ & $(0.0362)$ \\
\hline \multicolumn{6}{|l|}{5} \\
\hline IP & 4.0 & 7.0 & 0.25 & 1.00 & 1.00 \\
\hline PM & 3.9962 & 7.0126 & 0.2483 & 0.9840 & 0.9836 \\
\hline SD & $(0.0148)$ & $(0.0322)$ & $(0.0036)$ & $(0.0178)$ & (0.0198) \\
\hline \multicolumn{6}{|c|}{ 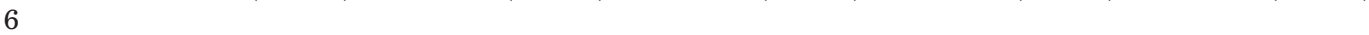 } \\
\hline IP & 4.0 & 7.0 & 0.10 & 1.00 & 1.00 \\
\hline PM & 3.9922 & 6.9834 & 0.1010 & 0.9911 & 1.0050 \\
\hline SD & $(0.0084)$ & $(0.0342)$ & $(0.0054)$ & $(0.0167)$ & $(0.0186)$ \\
\hline \multicolumn{6}{|l|}{7} \\
\hline IP & 4.0 & 7.0 & 0.50 & 1.00 & 1.00 \\
\hline PM & 4.0149 & 6.9960 & 0.4949 & 1.0287 & 1.0290 \\
\hline SD & $(0.0257)$ & $(0.0198)$ & $(0.0071)$ & $(0.0342)$ & $(0.0199)$ \\
\hline \multicolumn{6}{|l|}{8} \\
\hline IP & 4.0 & 6.0 & 0.25 & 1.00 & 1.00 \\
\hline PM & 4.0060 & 6.0085 & 0.2464 & 1.0083 & 1.0074 \\
\hline SD & (0.0359) & $(0.0439)$ & $(0.0159)$ & $(0.0203)$ & $(0.0207)$ \\
\hline \multicolumn{6}{|c|}{ 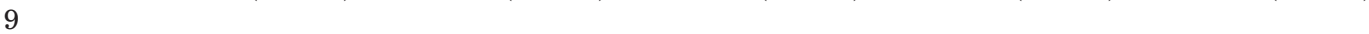 } \\
\hline IP & 4.0 & 8.0 & 0.25 & 1.00 & 1.00 \\
\hline PM & 4.0027 & 7.9900 & 0.2508 & 0.9979 & 1.0174 \\
\hline SD & $(0.0154)$ & $(0.0343)$ & $(0.0036)$ & $(0.0203)$ & $(0.0501)$ \\
\hline
\end{tabular}

was assumed to have the same distribution as the $\mathrm{PE}$ effects, but it included both additive genetic and PE components, ignoring relationship information. For model 1 , values of $k$ and $m$ were similar to those chosen

Table 2. Sensitivity, specificity, and probabilities of misclassification (PMC) in simulation I, averaged over 5 replicates. Sensitivity and specificity are defined as the probabilities of correct classification for observations from cows with health status IMI+ and IMI-, respectively.

\begin{tabular}{llll}
\hline Setting & Sensitivity & Specificity & PMC \\
\hline 1 & 0.9195 & 0.9829 & 0.0333 \\
2 & 0.7402 & 0.9611 & 0.0944 \\
3 & 0.5264 & 0.9439 & 0.1615 \\
4 & 0.8489 & 0.8738 & 0.1322 \\
5 & 0.8374 & 0.9479 & 0.0797 \\
6 & 0.7525 & 0.9711 & 0.0506 \\
7 & 0.8974 & 0.8979 & 0.1023 \\
8 & 0.6265 & 0.8807 & 0.1828 \\
9 & 0.9396 & 0.9803 & 0.0299 \\
\hline
\end{tabular}

in simulation I, while for model 2 , values of $k$ and $m$ were chosen to 10,000, and 50,000, respectively. For model $3, k$ and $m$ were 10,000 and 60,000 , respectively. Only samples where $\Delta>0$ were accepted.

\section{Model Evaluation}

For all models, each element of $\mathbf{z}\left(\mathrm{z}_{\mathrm{i}}\right)$ was drawn from a Bernoulli distribution with probability $\tau(\cdot)$, as indicated in [15]. After burn-in, the $\mathrm{z}_{\mathrm{i}}$ (considered to be draws from their marginal distributions) were averaged over Gibbs samples to obtain an estimate of the posterior probability of putative mastitis (PPM) for each observation.

Possible criteria for evaluating the mixture models were: 1) probability of misclassification (PMC), which is the probability of incorrect classification of health status averaged over rounds of the Gibbs sampler and 2) sensitivity and specificity of models. Sensitivity and 
specificity were defined as the probabilities of correct classification of IMI+ and IMI- cows, respectively. Sensitivity and specificity for cow $i$ is $\operatorname{Pr}\left(z_{i}=1 \mid t_{i}=1\right)$ and $\operatorname{Pr}\left(z_{i}=0 \mid t_{i}=0\right)$ respectively, where $t_{i}=\left\{\begin{array}{l}0 \text { if IMI }- \\ 1 \text { if IMI }+\end{array}\right.$. These parameters were estimated by averaging over rounds of the Gibbs sampler, and then computing:

$$
\mathrm{PMC}=1-\frac{\sum_{i=1}^{n}\left(\left(1-\mathrm{t}_{\mathrm{i}}\right)\left(1-\mathrm{PPM}_{\mathrm{i}}\right)+\mathrm{t}_{\mathrm{i}} \mathrm{PPM}_{\mathrm{i}}\right)}{n},
$$

$$
\begin{gathered}
\text { Sensitivity }=\frac{\sum_{i=1}^{n} \mathrm{t}_{\mathrm{i}} \mathrm{PPM}_{\mathrm{i}}}{\sum_{i=1}^{n} \text {, and }} \\
\text { Specificity }=\frac{\sum_{i=1}^{n}\left(1-\mathrm{t}_{\mathrm{i}}\right)\left(1-\mathrm{PPM}_{\mathrm{i}}\right)}{n-\sum_{i=1}^{n} \mathrm{t}_{\mathrm{i}}} .
\end{gathered}
$$

$\mathrm{PPM}_{\mathrm{i}}$ is the posterior probability of putative mastitis for observation $i$, and $n$ is the total number of observations.

\section{RESULTS AND DISCUSSION}

\section{Simulation I}

The results from the analyses of simulation I are shown in Table 1. For homogeneous residual variance (settings 5 to 9), input parameters were estimated essentially without bias. On the other hand, when heterogeneous residual variances were simulated and fitted (settings 1 to 4 ), estimates seemed to be systematically biased. Identifying the components and their corresponding residual variances seemed difficult in this case. Estimates of residual variances for IMI- and IMI+ tended towards each other, with the largest effect on the estimates of variance in the smallest group (IMI+). Seemingly biased estimates of the mixing proportion and of location parameters were observed also. The simulations with the most overlapping mixture components (setting 3) also had the largest bias. The bias of the residual variances may be reduced via use of more informative priors.

Sensitivity, specificity, and PMC for the different settings of simulation I are presented in Table 2. Sensitivity ranged between 0.53 (setting 3) and 0.94 (setting 9), whereas specificity varied between 0.87 (setting 4) and 0.98 (setting 1). Except for setting 7, sensitivity was lower than specificity, which could be explained by a $P_{m}$ lower than 0.5 for those settings, indicating a lower probability of IMI+ than IMI-. The results suggest that for simulations with $P_{m}<0.5$, the model identifies healthy animals with little error (high specificity), but that up to almost $50 \%$ of the diseased animals can be misclassified (setting 3). Overall, PMC ranged between 0.03 (settings 1 and 9 ) and 0.18 (setting 8 ). There was no clear relationship between the quality of the parameter estimates and the sensitivity, specificity, or PMC. As expected, the settings with the most overlapping mixture components (3 and 8) had the largest PMC.

\section{Simulation II}

Recall that in model 1 genetic and PE effects were ignored; model 2 fitted genetic and $\mathrm{PE}$ deviations as a cow effect (ignoring relationships), and model 3 accounted for both genetic (including relationships) and $\mathrm{PE}$ effects. Hence, in model 1 the residual variance is expected to be equal to the sum of all variance components, while in model 2 the variance of cow effects is expected to correspond to the sum of additive genetic and PE variance components. Posterior means of the parameters in the three models, averaged over five replicates, are presented in Table 3. Location and dispersion parameters and $P_{m}$ were returned with a high degree of accuracy, irrespective of whether or not the random effects were combined or modeled properly.

When random effects were fitted, sensitivity and specificity increased substantially relative to the fixed model, whereas PMC decreased (Table 4). As expected, the reduction in $\mathrm{PMC}$ via introduction of random effects was largest in the simulations with the largest genetic and PE variances (setting 2). There were negligible differences in sensitivity, specificity, and PMC between those models including a cow effect without using relationship information and those where both additive genetic and PE effects were fitted. Hence, a model using a single random factor accounting for animal effects seemed to classify observations with the same precision as the more complex model. In simulations with a different data structure, e.g., fewer records per cow and larger daughter groups per sire, inclusion of genetic effects may have a relatively greater impact on PMC.

Although model 2 and model 3 classified observations with the same sensitivity and specificity, the latter had smaller variance between replicates in posterior means of the fixed effects $\left(\mu_{0}\right.$ and $\left.\mu_{1}\right)$ (Table 3). A plausible explanation is that model 3 allows accounting for genetic drift, which may randomly change genetic level of SCS over generations. Since genetic drift is a random process, its effect on estimates of fixed effects is averaged out over replicated simulations. In practice, due to selection, the genetic level of SCS may change more rapidly and in a specific direction. Hence, including 
Table 3. Input parameters (IP) for two settings in simulation II and their posterior means (PM) from three mixture models, averaged over 5 replicates. Standard deviations (SD) of PM from the 5 replicates are placed in brackets. Parameters for simulated SCS are: mean of cows with health status IMI- $\left(\mu_{0}\right)$ and IMI+ $\left(\mu_{1}\right)$, mixing proportion $\left(\mathrm{P}_{m}\right)$, variance between cows $\left(\sigma_{c}^{2}\right)$, variance of permanent environmental effects $\left(\sigma_{p}^{2}\right)$, additive genetic variance $\left(\sigma_{a}^{2}\right)$, and the respective residual variances for cows with IMI- and IMI+ $\left(\sigma_{e 0}^{2}\right.$ and $\left.\sigma_{e 1}^{2}\right)$.

\begin{tabular}{|c|c|c|c|c|c|c|c|c|c|c|}
\hline Setting & Model & & $\mu_{0}$ & $\mu_{1}$ & $P_{m}$ & $\sigma_{c}^{2}$ & $\sigma_{p}^{2}$ & $\sigma_{a}^{2}$ & $\sigma_{e 0}^{2}$ & $\sigma_{e 1}^{2}$ \\
\hline \multirow[t]{7}{*}{1} & & IP & 4.00 & 6.00 & 0.25 & $\cdots$ & 0.30 & 0.10 & 0.60 & 0.60 \\
\hline & \multirow[t]{2}{*}{1} & PM & 3.9771 & 5.9971 & 0.2483 & . . & $\ldots$ & $\ldots$ & 1.0039 & 1.0024 \\
\hline & & $\mathrm{SD}$ & $(0.0360)$ & $(0.0413)$ & $(0.0064)$ & & & & $(0.0158)$ & $(0.0150)$ \\
\hline & \multirow[t]{2}{*}{2} & PM & 3.9760 & 5.9801 & 0.2509 & 0.4031 & . & . & 0.6038 & 0.6034 \\
\hline & & SD & $(0.0360)$ & $(0.0463)$ & $(0.0034)$ & $(0.0087)$ & & & $(0.0085)$ & $(0.0053)$ \\
\hline & \multirow[t]{2}{*}{3} & PM & 4.0051 & 6.0090 & 0.2510 & $\ldots$ & 0.2953 & 0.1061 & 0.6037 & 0.6032 \\
\hline & & SD & $(0.0241)$ & $(0.0200)$ & $(0.0036)$ & $\ldots$ & $(0.0179)$ & $(0.0209)$ & $(0.0083)$ & $(0.0054)$ \\
\hline \multirow[t]{7}{*}{2} & & IP & 4.00 & 6.00 & 0.25 & $\ldots$ & 0.40 & 0.20 & 0.40 & 0.40 \\
\hline & \multirow[t]{2}{*}{1} & $\mathrm{PM}$ & 4.0235 & 5.9962 & 0.2485 & $\ldots$ & $\ldots$ & $\ldots$ & 1.0215 & 1.0204 \\
\hline & & SD & $(0.0456)$ & $(0.0494)$ & $(0.0061)$ & & & & $(0.0119)$ & $(0.0118)$ \\
\hline & \multirow[t]{2}{*}{2} & PM & 4.0141 & 6.0116 & 0.2501 & 0.6061 & $\ldots$ & $\ldots$ & 0.4009 & 0.3991 \\
\hline & & SD & $(0.0397)$ & $(0.0428)$ & $(0.0033)$ & $(0.0210)$ & $\ldots$ & $\ldots$ & $(0.0022)$ & $(0.0052)$ \\
\hline & \multirow[t]{2}{*}{3} & PM & 4.0157 & 6.0130 & 0.2502 & $\ldots$ & 0.3951 & 0.2067 & 0.4009 & 0.3991 \\
\hline & & $\mathrm{SD}$ & $(0.0298)$ & $(0.0288)$ & $(0.0034)$ & $\ldots$ & $(0.0169)$ & $(0.0333)$ & $(0.0021)$ & $(0.0055)$ \\
\hline
\end{tabular}

genetic effects in the model may improve inferences, although no effect on PMC was observed for our simulations.

\section{General Discussion}

In our simulations, random effects were assigned identical distributions across mixture components, while in real data the covariance structure may be more complex. To account for this, the mixture model could be extended to allow for a covariance between random effects in the different mixture components. Using such models, breeding values for SCS response to udder infection could be predicted. Additionally, multivariate information could be incorporated in the analysis. Traits that could be potentially regarded as mixtures, depending on the cows' udder health status, are electrical conductivity in milk and even test-day milk production. Adding traits to the analysis may yield additional information about the cows' udder health status, thus increasing the probability of correct classification. Real SCS data may represent more than two groups (e.g.,

Table 4. Sensitivity, specificity, and probabilities of misclassification (PMC) from simulation II, averaged over 5 replicates. Sensitivity and specificity are defined as the probabilities of correct classification for observations of cows with health status IMI+ and IMI-, respectively.

\begin{tabular}{lllll}
\hline Setting & Model & Sensitivity & Specificity & PMC \\
\hline 1 & 1 & 0.6315 & 0.8802 & 0.1822 \\
& 2 & 0.7280 & 0.9092 & 0.1363 \\
& 3 & 0.7288 & 0.9093 & 0.1360 \\
2 & 1 & 0.6231 & 0.8765 & 0.1869 \\
& 2 & 0.8228 & 0.9410 & 0.0886 \\
& 3 & 0.8234 & 0.9410 & 0.0884 \\
\hline
\end{tabular}

healthy, clinical mastitis, and subclinical mastitis) so it would be desirable to develop mixture models with more than two components.

Individual PPM from a mixture model might be a competing alternative to EBV for crude SCS in selection against susceptibility to mastitis. In this study, individual elements of the classification vector $(\mathbf{z})$ were assumed independent a priori, and following the same Bernoulli distribution, implying identical prior probability of mastitis for all cows. However, genetic and PE effects on liability to mastitis have been reported (e.g., Heringstad et al., 2003), causing differing probabilities of mastitis between and within families. Hence, genetic selection based on PPM from a mixture model assuming a priori identical probabilities of mastitis for all observations might lead to distorted inferences. A challenge is to develop hierarchical mixture models that make use of family-specific and cow-specific putative liability to mastitis in estimation of the posterior probability of mastitis for each observation, instead of a single $P_{m}$, as in equation [15].

\section{CONCLUSIONS}

Results from mixture model analyses of simulated SCS were encouraging. When the residual variance was the same in the two mixture components, simulated parameters were estimated with high precision; however, some bias occurred in simulations with unequal residual variances. When the mixture components overlapped, residual variances were difficult to distinguish from each other; this was seemingly associated with overestimation of the proportion of the mixture component with the smallest residual variance and corres- 
ponding underestimation of the other component. When random factors common to both mixture components affected SCS, inclusion of such factors in the models increased the probability of correct classification. Separation of animal effects into genetic and nongenetic components had a negligible effect on the probability of correct classification. However, the variance between posterior mean estimates of fixed effects in the different replicates was smaller, as the model accounts for changes in the genetic level of SCS caused by genetic drift. Hence, including genetic effects in the model might be important, especially when genetic change is directional.

\section{ACKNOWLEDGMENTS}

The project has received funding from the Research Council of Norway, TINE Norwegian Dairies BA, GENO Breeding and A.I. Association, and The Nordic Academy for Advanced Study (NorFA). Daniel Gianola wishes to acknowledge support from grants NRICGP/ USDA 99-35205-8162, NRICGP/USDA 2003-3520512833, and NSF DEB-0089742.

\section{REFERENCES}

Casella, G., and E. I. George. 1992. Explaining the Gibbs sampler. Am. Stat. 46:167-174.

Detilleux, J., and P. L. Leroy. 2000. Application of a mixed normal mixture model for the estimation of mastitis-related parameters. J. Dairy Sci. 83:2341-2349.

Heringstad, B., Y. M. Chang, D. Gianola, and G. Klemetsdal. 2003. Genetic analysis of longitudinal trajectory of clinical mastitis in first-lactation Norwegian Cattle. J. Dairy Sci. 86:2676-2683.
Jansen, R. C. 1992. A general mixture model for mapping quantitative trait loci by using molecular markers. Theor. Appl. Genet. 85:252-260.

Kao, C. H., and Z. B. Zeng. 1997. General formulas for obtaining the MLEs and the asymptotic variance-covariance matrix in mapping quantitative trait loci when using the EM algorithm. Biometrics 53:653-665.

Kuhn, M. T., A. E. Freeman, and R. L. Fernando. 1999. Approaches investigated to correct for preferential treatment. J. Dairy Sci. 82:181-190.

McLachlan, G., and D. Peel. 2000. Finite Mixture Models. John Wiley \& Sons, Inc., New York, NY.

Militino, A. F., M. D. Ugarte, and C. B. Dean. 2001. The use of mixture models for identifying high risks in disease mapping. Stat. Med. 20:2035-2049.

Mrode, R. A., and G. J. T. Swanson. 1996. Genetic and statistical properties of somatic cell count and its suitability as an indirect means of reducing the incidence of mastitis in dairy cattle. Anim. Breed. Abstr. 64:847-857.

Raftery, A. L., and S. Lewis. 1992. How many iterations in the Gibbs sampler? Pages 763-774 in Bayesian Statistics 4. J. M. Bernando, J. O. Berger, A. P. Dawid, and A. F. M. Smith, ed. Oxford University Press, Oxford, UK.

Schukken, Y. H., B. A. Mallard, J. C. M. Dekkers, K. E. Leslie, and M. J. Stear. 1994. Genetic impact on the risk of intramammary infection following Staphylococcus aureus challenge. J. Dairy Sci. 77:639-647.

Schukken, Y. H., K. E. Leslie, D. A. Barnum, B. A. Mallard, J. H. Lumsden, P. C. Dick, G. H. Vessie, and M. E. Kehrli. 1999. Experimental Staphylococcus aureus intramammary challenge in late lactation dairy cows: Quarter and cow effects determining the probability of infection. J. Dairy Sci. 82:2393-2401.

Shuster, D. E., E. K. Lee, and M. E. Kehrli. 1996. Bacterial growth, inflammatory cytokine production, and neutrophil recruitment during coliform mastitis in cows within ten days after calving, compared with cows at midlactation. Am. J. Vet. Res. 57:1569 1575 .

Sorensen, D., and D. Gianola. 2002. Likelihood, Bayesian, and MCMC Methods in Quantitative Genetics. Springer-Verlag, New York, NY.

Suriyasathaporn, W., Y. H. Schukken, M. Nielen, and A. Brand. 2000. Low somatic cell count: A risk factor for subsequent clinical mastitis in a dairy herd. J. Dairy Sci. 83:1248-1255. 